\title{
The E3 ubiquitin ligase specificity subunit ASB2 $\beta$ is a novel regulator of muscle differentiation that targets filamin B to proteasomal degradation
}

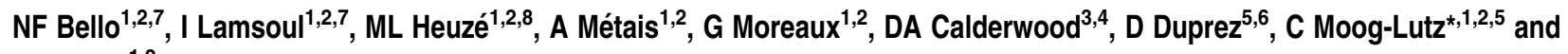 \\ PG Lutz ${ }^{\star, 1,2}$
}

\begin{abstract}
Ubiquitin-mediated protein degradation is the main mechanism for controlled proteolysis, which is crucial for muscle development and maintenance. The ankyrin repeat-containing protein with a suppressor of cytokine signaling box 2 gene (ASB2) encodes the specificity subunit of an E3 ubiquitin ligase complex involved in differentiation of hematopoietic cells. Here, we provide the first evidence that a novel ASB2 isoform, ASB2 $\beta$, is important for muscle differentiation. ASB2 $\beta$ is expressed in muscle cells during embryogenesis and in adult tissues. ASB2 $\beta$ is part of an active E3 ubiquitin ligase complex and targets the actin-binding protein filamin B (FLNb) for proteasomal degradation. Thus, ASB2 $\beta$ regulates FLNb functions by controlling its degradation. Knockdown of endogenous ASB2 $\beta$ by shRNAs during induced differentiation of $\mathrm{C}_{2} \mathrm{C} 12$ cells delayed FLNb degradation as well as myoblast fusion and expression of muscle contractile proteins. Finally, knockdown of FLNb in ASB2 $\beta$ knockdown cells restores myogenic differentiation. Altogether, our results suggest that ASB2 $\beta$ is involved in muscle differentiation through the targeting of FLNb to destruction by the proteasome.
\end{abstract}

Cell Death and Differentiation (2009) 16, 921-932; doi:10.1038/cdd.2009.27; published online 20 March 2009

The ubiquitin-proteasome system (UPS) is one of the major mechanisms for controlled proteolysis, which is a crucial determinant of many cellular events in eukaryotes. Degradation of a protein by the ubiquitin-proteasome pathway entails two successive events: the covalent attachment of ubiquitin chains to lysine residues in a substrate protein leading to its recognition and ATP-dependent proteolysis by the proteasome. Ubiquitylation of protein substrates occurs through the sequential action of distinct enzymes: a ubiquitin-activating enzyme E1, a ubiquitin-conjugating enzyme E2 and a ubiquitin ligase E3 responsible for the specific recognition of substrates through dedicated interaction domains. ${ }^{1}$ ASB2 is one of 18 members of the ankyrin repeat-containing suppressor of cytokine signaling (SOCS) box protein family (ASB) that are characterized by variable numbers of $\mathrm{N}$-terminal ankyrin repeats. ${ }^{2}$ The ASB2 gene was originally identified as an retinoic acid-inducible gene involved in induced differentiation of myeloid leukemia cells. ${ }^{3,4}$ We have previously demonstrated that, by interacting with the elongin BC complex, ASB2 can assemble with a Cullin5/Rbx module to form an E3 ubiquitin ligase complex that stimulates polyubiquitylation by the E2 ubiquitin-conjugating enzyme UbcH5a. ${ }^{5,6}$ This strongly suggests that ASB2 targets specific proteins to destruction by the proteasome during differentiation of hematopoietic cells. We have recently shown that ASB2 ubiquitin ligase activity drives proteasome-mediated degradation of the ubiquitously expressed actin-binding protein filamins (FLNs), FLNa and b, and can regulate integrin-mediated cell spreading. ${ }^{6}$

During muscle development, dramatic changes in protein expression and cell morphology rely on the turnover of regulatory and structural components. Indeed, myogenic transcription factors such as MyoD and its E2A partner or negative Id regulator as well as myofibrillar proteins were shown to be degraded by the UPS. ${ }^{7-11}$ Although some E3 ubiquitin ligases active during myogenesis have been identified, ${ }^{12-23}$ a precise understanding of the function of ubiquitylation in muscle development and the identities of specific ubiquitin ligases and their potential substrates is lacking.

Here we show that $A S B 2$ expression is not restricted to hematopoietic cells but is also expressed and regulated in muscle cells during mouse and chick embryogenesis.

\footnotetext{
${ }^{1}$ Institut de Pharmacologie et de Biologie Structurale, CNRS, 205 route de Narbonne, F-31077 Toulouse, France; ${ }^{2}$ Université de Toulouse, UPS, Institut de Pharmacologie et de Biologie Structurale, F-31077 Toulouse, France; ${ }^{3}$ Department of Pharmacology, Yale University School of Medicine, New Haven, CT 06520, USA; ${ }^{4}$ Interdepartmental Program in Vascular Biology and Transplantation, Yale University School of Medicine, New Haven, CT 06520, USA; ${ }^{5}$ Université Pierre et Marie Curie, Paris, France and ${ }^{6} \mathrm{CNRS}$, UMR 7622, Paris, France

${ }^{*}$ Corresponding authors: PG Lutz or C Moog-Lutz, Institut de Pharmacologie et de Biologie Structurale, UMR 5089 CNRS, 205 Route de Narbonne, F-31077 Toulouse, France. Tel: + 33561175 471; Fax: + 33561175 994. E-mails: Pierre.Lutz@ipbs.fr, Christel.Lutz@ipbs.fr

${ }^{7}$ These authors contributed equally to this work.

${ }^{8}$ Current address: INSERM U653, Institut Curie, Paris, France.

Keywords: ubiquitin-proteasome system; $A S B 2$; muscle differentiation; filamin

Abbreviations: ASB2, ankyrin repeat-containing protein with a suppressor of cytokine signaling box 2 gene; CRL, Cullin RING ligase; FLN, filamin; HECT, homologous to the E6-associated protein C terminus; MHC, myosin heavy chain; MURF, muscle RING finger; RING, really interesting new gene; SOCS, suppressor of cytokine signaling; UIM, ubiquitin-interacting motif; UPS, ubiquitin-proteasome system

Received 26.8.08; revised 29.1.09; accepted 10.2.09; Edited by G Cossu; published online 20.3.09
} 
Furthermore, ASB2 transcripts expressed in muscle cells encode for a novel ASB2 isoform that we have named ASB2 $\beta$. Its expression is induced during myogenic differentiation of $\mathrm{C} 2 \mathrm{C} 12$ and primary myoblasts. By interacting with the elongin BC complex, ASB2 $\beta$ can assemble with the Cullin5/Rbx2 module to reconstitute an active E3 ubiquitin ligase complex and we show that ASB2 $\beta$ triggers ubiquitylation and drives proteasomal degradation of FLNb but not of FLNa. Knockdown of ASB2 $\beta$ expression markedly delayed FLNb degradation and decreased $\mathrm{C} 2 \mathrm{C} 12$ differentiation. Thus, our study provides the first evidence that FLNb regulation, through proteasomal degradation pathways, may regulate muscle differentiation.

\section{Results}

ASB2 is expressed in developing and mature muscle cells. ASB2 is known to be expressed in hematopoietic cells, where it is important for cell differentiation. ${ }^{3,6}$ To examine whether ASB2 may have functions outside the hematopoietic system, we examined expression of ASB2 mRNA in a variety of other tissues. Human ASB2 mRNA was detected in bone marrow, skeletal muscle, heart, fetal heart, small intestine, appendix, bladder, aorta, stomach, uterus, prostate, colon and thyroid gland (Figure 1a). Human ASB2 transcripts were relatively less abundant in tissues containing nonstriated muscle than in skeletal and cardiac muscle. ASB2 expression was induced during mouse embryonic development (Figure 1b) and its expression was maintained in skeletal muscle and heart in the adult mice (Figure 1c). To examine the expression of ASB2 during chick embryogenesis, we performed in situ hybridization to whole-mount embryos and to tissue sections at various stages of development (Figure 2). In situ hybridization to whole-mount embryos showed an expression of ASB2 in the somites (Figure $2 \mathrm{a}-\mathrm{c}$ and $\mathrm{e}$ ), and in the heart (Figure $2 \mathrm{c}$ and g) from E3 and in the limb (Figure 2d) from E6. In situ hybridization to tissue sections showed that the somitic expression of $A S B 2$ corresponds to the myotome (Figure 2e). ASB2 transcripts are detected in all the heart (Figure 2g). An additional site of $A S B 2$ expression was observed in smooth muscle cells of the intestine at E6 (Figure 2f). Finally, the limb ASB2 expression corresponds to skeletal muscle expression (Figure $2 \mathrm{~d}$ and h). Altogether these results demonstrated that $A S B 2$ is developmentally regulated and that its expression previously described in hematopoiesis is also associated with the formation of all types of muscles, including skeletal, smooth and cardiac muscles.

ASB2 is induced during myogenic differentiation. Because myogenic differentiation can be recapitulated in vitro, wherein myoblasts can be converted to myotubes, we examined the expression of ASB2 mRNA throughout the well-established differentiation model of the $\mathrm{C} 2 \mathrm{C} 12$ mouse cell line. Differentiation of $\mathrm{C} 2 \mathrm{C} 12$ cells was confirmed by their morphological changes such as alignment, elongation and fusion of mononucleated cells to multinucleated myotubes after switching cells from growth medium (GM) to differentiation

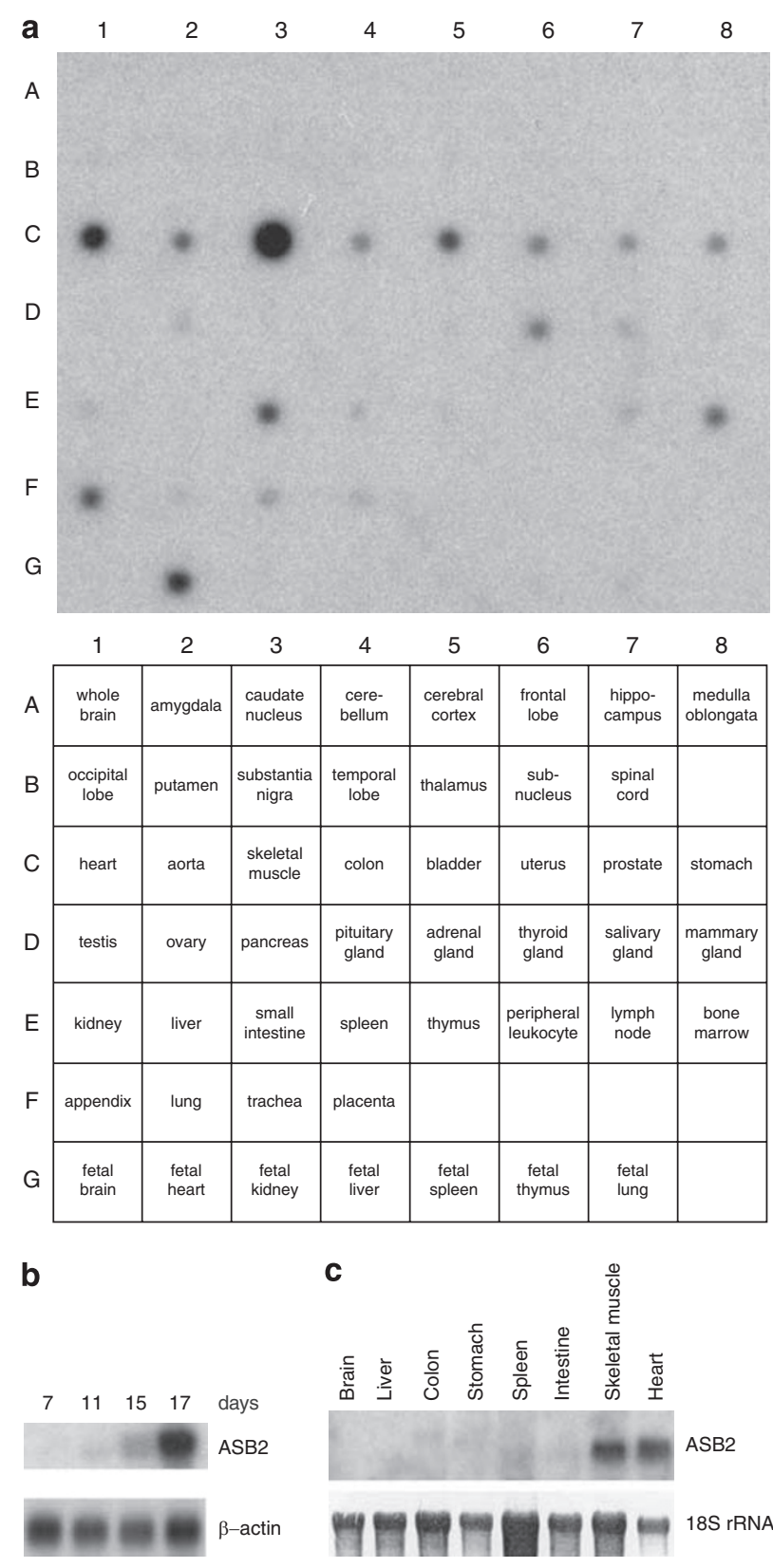

Figure 1 Expression of $A S B 2 \mathrm{mRNA}$ in tissues. (a) Autoradiogram of ASB2 mRNA expression in human primary tissues (upper) and diagram showing the type and position of polyA + RNAs on the membrane (lower). Autoradiograms of ASB2 mRNAs expression in mouse embryos (b) and in mouse adult tissues (c). Northern blot was performed using $2 \mu \mathrm{g}$ of polyA + RNA (b) or $5 \mu \mathrm{g}$ of total RNA (c). To confirm RNA loading and integrity, we used $\beta$-actin as a probe (b) or methylene blue-stained $18 \mathrm{~S}$ rRNA on membrane after transfer (c)

medium (DM) (Figure 3a). Accompanying these morphological changes, the expression of muscle-specific proteins, myogenin, myosin heavy chain (MHC) and troponin $\mathrm{T}$ was upregulated (Figure $3 b$ and $c$ ). The ASB2 transcripts were barely detectable in undifferentiated $\mathrm{C} 2 \mathrm{C} 12$ cells, increased in cells cultured in $\mathrm{DM}$ for $8 \mathrm{~h}$ and were continuously expressed until day 8 (Figure 3d). By this time, myogenin, an early marker for the entry of myoblast into the differentiation, was induced 

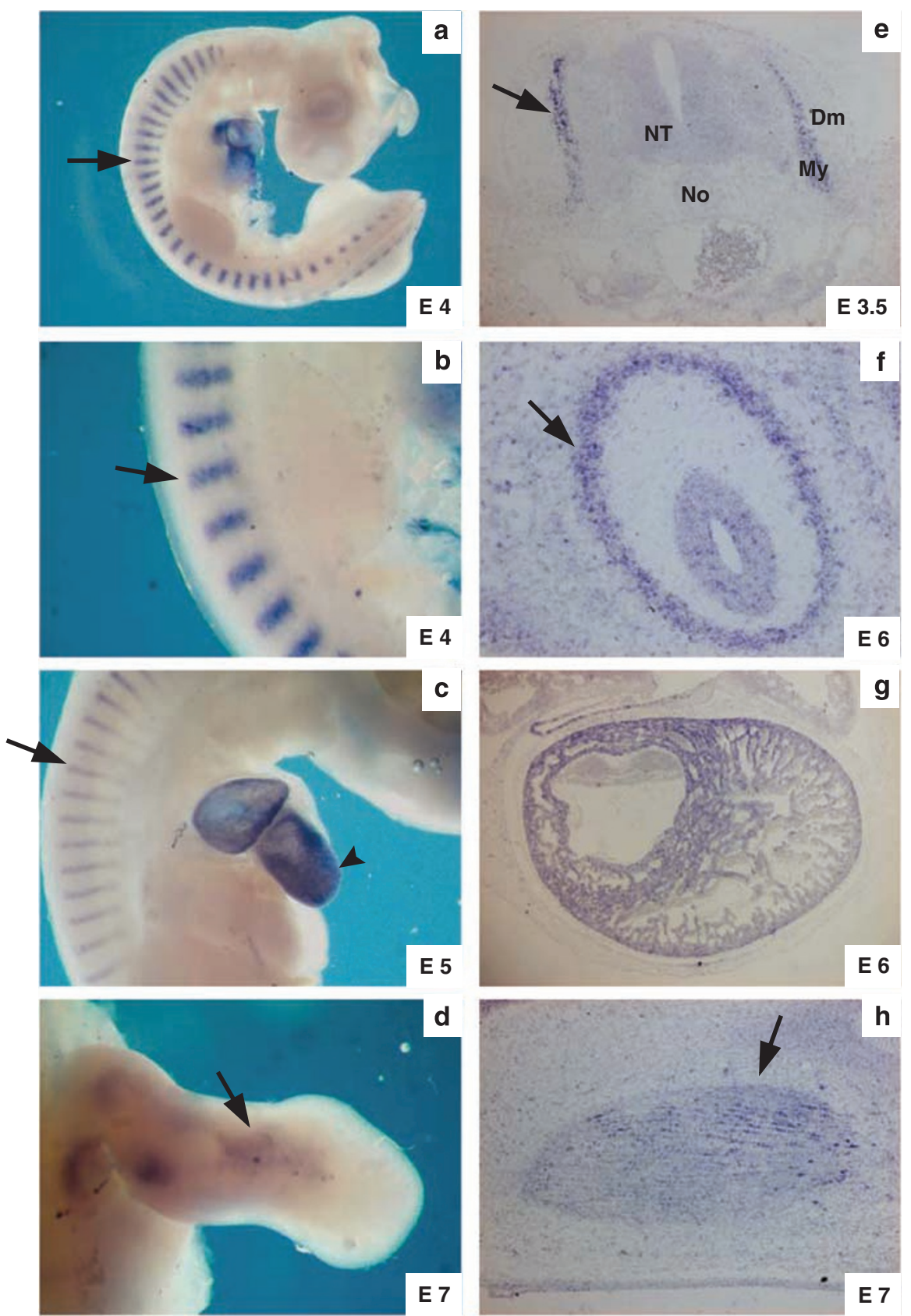

Figure 2 Expression pattern of ASB2 during chick embryogenesis. ASB2 mRNAs were detected in whole-mount preparations (a-d) and sections through the trunk region (e), the intestine (f), the heart $(\mathbf{g})$ or the developing limb skeletal muscle $(\mathbf{g})$ of chick embryos by in situ hybridization. In each panel, the developmental stage is indicated. NT, neural tube; No, notochord; Dm, dermomyotome; My, myotome. Arrows indicate somites in a-c and $\mathbf{e}$, limb skeletal muscle in $\mathbf{d}$ and $\mathbf{h}$, and smooth muscle cells of the intestine in $\mathrm{f}$. Arrowhead points to the heart

(Figure $3 b$ ), suggesting that $A S B 2$ upregulation may coincide with the differentiation commitment of myoblasts. Altogether, our results show that $A S B 2$ is induced during myogenic differentiation.

ASB2 $\beta$, a novel ASB2 isoform. The cDNA sequences encoding mouse ASB2 proteins were first analyzed in EST databases. Two different isoforms, a hematopoietic type and a muscle type, of ASB2 were identified and named ASB2 $\alpha$ and ASB2 $\beta$, respectively (Figure $4 a$ ). The human ASB2 $\alpha$ has been previously published. ${ }^{3,5}$ The mouse ASB2 $\alpha$ was recently described in UniProtKB/Swiss-Prot database (release 12.0/54.0) as isoform 2 of Q8KOL0 whereas mouse ASB2 $\beta$ corresponds to isoform 1 of Q8KOL0. To confirm the expression of two ASB2 mRNAs, we performed quantitative RT-PCR experiments by amplification of CDNAs from skeletal muscle, heart, smooth muscle and hematopoietic cells with primers specific to ASB2 $\alpha$, ASB2 $\beta$ or with primers common to both cDNAs. As shown in Figure $4 \mathrm{~b}, \mathrm{ASB} 2 \beta$ mRNAs were mainly expressed in muscle 
cells whereas ASB2 $\alpha$ mRNAs were expressed in hematopoietic cells. The new $\beta$ isoform of ASB2 retains ankyrin repeats and the SOCS box (Figure 4a). In addition, ASB2 $\beta$ harbors a ubiquitin-interacting motif (UIM) at its $N$ terminus (Figure 4a and b). The ASB2 SOCS box can be further divided into a $\mathrm{BC}$ box that defines a binding site for the elongin $\mathrm{BC}$ complex and a Cullin5 box that determines the binding specificity for Cullin $5^{5,24}$ (Figure 4c). ASB2 $\alpha$ and ASB2 $\beta$ are predicted to have molecular weights of 64 and $70 \mathrm{kDa}$, respectively (Figure $4 \mathrm{a}$ ). To extend and further validate the finding that the ASB2 $\beta$ isoform is expressed in muscle cells, we raised anti-peptide polyclonal antibodies against an epitope within the $\mathrm{N}$-terminal extension of ASB2 $\beta$ (2PNAB1 serum) or against an epitope within the $C$ terminus common to both ASB2 isoforms (1PLA serum) (Figure 4a). Flag-tagged ASB2 isoforms were expressed in HeLa cells to test the specificity of the antibodies by western blot analysis. As expected, anti-ASB2 antibodies from the 1PLA serum recognized both $A S B 2 \alpha$ and $A S B 2 \beta$ isoforms whereas the anti-ASB2 $\beta$ antibodies from the 2PNAB1 serum recognized only the ASB2 $\beta$ isoform (Figure $4 d$ ). In protein lysates from differentiating $\mathrm{C} 2 \mathrm{C} 12$ cells, a $70 \mathrm{kDa}$ band was detected by

a

day 0

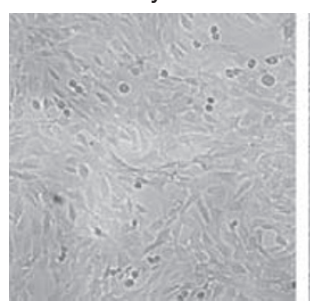

b

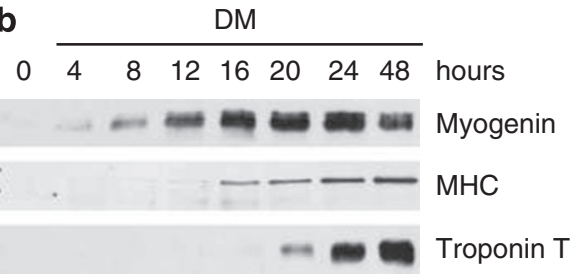

day 2

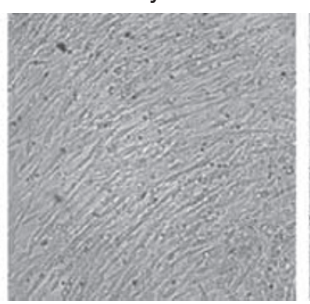

C
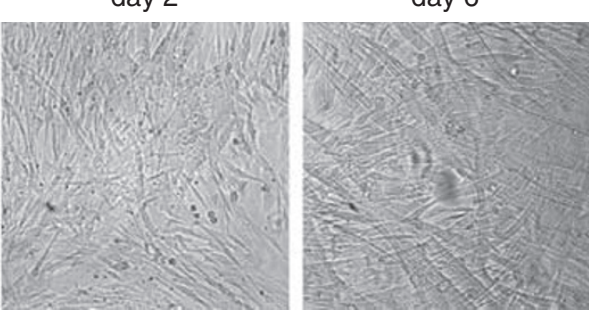

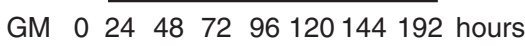

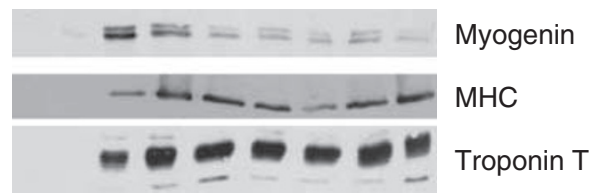

DM

d

$48 \quad 72 \quad 96 \quad 120144192$ hours

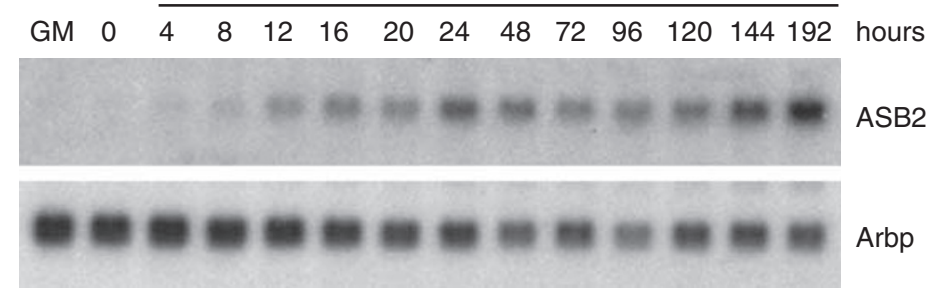

Figure 3 Induction of ASB2 mRNA during differentiation of $\mathrm{C} 2 \mathrm{C} 12$ myoblasts. $\mathrm{C} 2 \mathrm{C} 12$ cells were cultured in growth medium (GM) and shifted to differentiation medium (DM) for 6 days. (a) Morphological changes of $\mathrm{C} 2 \mathrm{C} 12$ cells for assessment of alignment, elongation and fusion were observed under a phase-contrast microscope. (b and $\mathbf{c}$ ) Expression of myogenin, myosin heavy chain ( $\mathrm{MHC}$ ) and troponin T during differentiation of $\mathrm{C} 2 \mathrm{C} 12$ cells. Aliquots $(10 \mu \mathrm{g})$ of each whole-cell extract were analyzed by western blot using indicated antibodies. (d) Detection of ASB2 mRNA in C2C12 cells cultured in GM or in DM for 1-6 days. Northern blot analysis was performed using $5 \mu \mathrm{g}$ of total RNA. Upper and lower panels are autoradiograms of mRNA of ASB2 and Arbp as assessment of RNA quantities in each lane, respectively

\footnotetext{
Figure 4 Characterization of ASB2 isoforms. (a) Schematic representation of the domains of mouse ASB2 isoforms. Positions of the peptide sequences used for production of the 2PNAB1 and 1PLA sera are shown. (b) Relative expression of ASB2 mRNAs in human skeletal muscle, heart, smooth muscle and myeloid cells. Quantitative real-time RT-PCR was carried out as described in Materials and Methods section. Results are plotted as relative expression for ASB2 $\alpha$ and ASB2 $\beta$. Data corresponding to one out of three independent experiments are shown as mean \pm standard deviation. (c) Alignments of the ubiquitin-interacting motif (UIM), the BC box and the Cullin5 box of mouse ASB2 $\beta$ with the consensus sequences. Shaded regions represent residues identical (black) or similar (gray) to the domain class consensus sequences. e is a negatively charged residue, $\Phi$ represents hydrophobic residue and $\mathrm{x}$ is any amino acid. In $\mathbf{a}$ and $\mathbf{b}$, amino-acid numbering is indicated. (d) Isoform specificity of ASB2-specific antibodies. HeLa cells were transfected with Flag-tagged ASB2 $\beta$ and ASB2 $\alpha$ expression vectors or the corresponding empty vector $(-)$. Urea soluble fractions $(5 \mu \mathrm{g})$ were separated by SDS-PAGE and subjected to immunoblotting with anti-Flag antibodies and 1PLA or 2PNAB1 serum, as indicated. (e) ASB2 $\beta$ protein is induced during differentiation of $\mathrm{C} 2 \mathrm{C} 12$ myoblasts. $\mathrm{C} 2 \mathrm{C} 12$ cells were cultured in $\mathrm{GM}$ and were shifted to $\mathrm{DM}$ for 2 and 4 days. Aliquots $(30 \mu \mathrm{g})$ of each urea soluble fraction were analyzed by western blot using the 2PNAB1 serum. (f) ASB2 $\beta$ associated with elongins B and C, Cullin5 and Rbx2. Sf21 cells were infected with baculoviruses expressing the proteins indicated. The lysates were immunoprecipitated (IP) with anti-Flag antibodies. Crude extracts (input) and immune complexes were separated by SDS-PAGE and immunoblotted with the indicated antibodies. ASB2 isoforms were detected using the 1PLA serum. ( $\mathbf{g}$ and $\mathbf{h}$ ) The ASB2 $\beta /$ elongin BC/Cullin5/Rbx2 complex had ubiquitin ligase activity. The cell lysates of $\mathbf{f}$ were subjected to anti-Flag immunoaffinity purification. The purified ASB2 complexes were incubated with Uba1, UbcH5a, ubiquitin and ATP to assess their ability to stimulate ubiquitylation by $\mathrm{UbcH} 5$ a by western blot using anti-polyubiquitin (g), anti-Flag ( $\mathbf{h}$, left panel) and anti-HA (h, right panel) antibodies. Arrow indicates the heavy chain of immunoglobulins $(\lg (\mathrm{H}))$
} 
the 2PNAB1 serum (Figure 4e), indicating that ASB2 $\beta$ protein is induced during skeletal muscle differentiation.

ASB2 $\beta$ is the specificity subunit of an E3 ubiquitin ligase complex. Given that ASB2 $\alpha$ is the specificity subunit of an E3 ubiquitin ligase complex and that ASB2 $\beta$ contains both
$\mathrm{BC}$ and Cullin5 boxes, we determined whether ASB2 $\beta$ can also assemble with elongin $B$, elongin $C$ and a Cullin5/Rbx module to reconstitute an E3 ubiquitin ligase complex. Therefore, anti-Flag immunoprecipitations were carried out on lysates of Sf21 cells coinfected with baculoviruses encoding Flag-ASB2 $\beta$, elongin B, HSV-elongin C, Rbx2
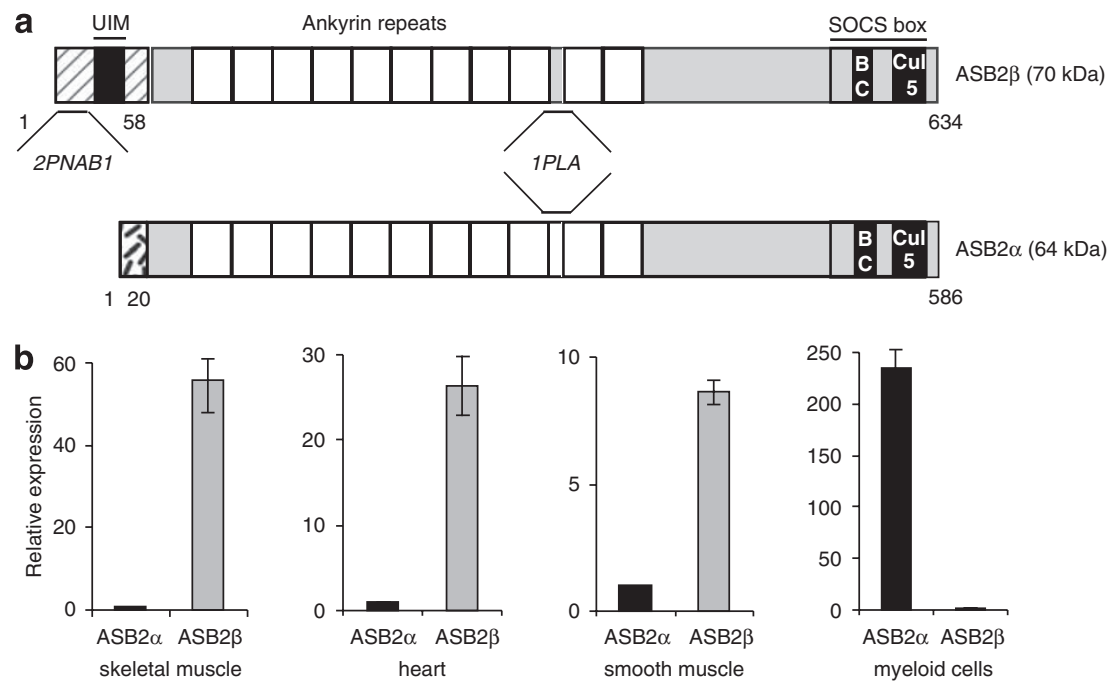

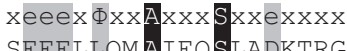
SEEELLQMAIEQSLADKTRG 26 UIM

d

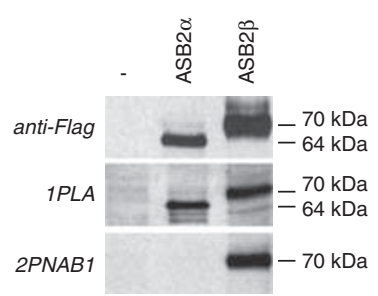

e

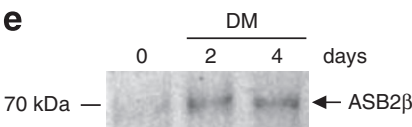

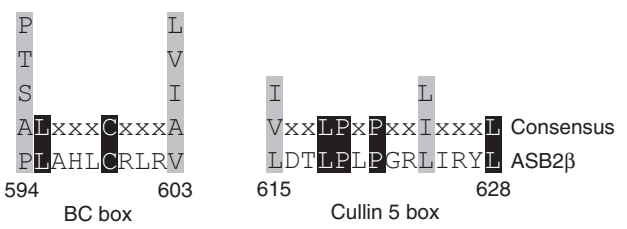
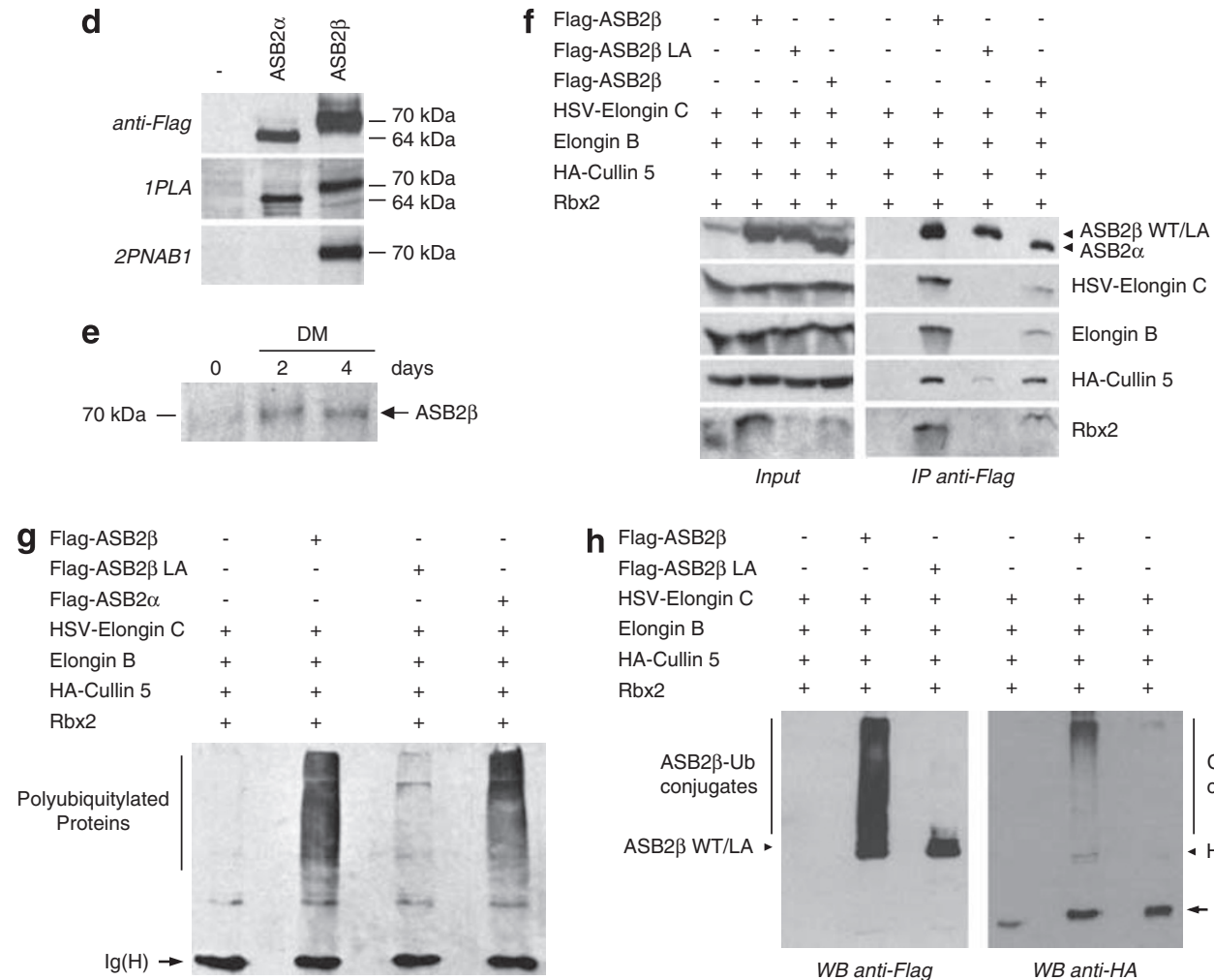

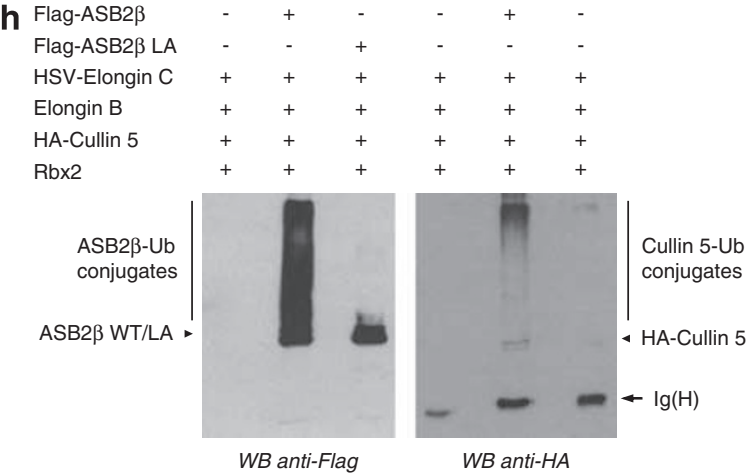


and HA-Cullin5, as indicated (Figure 4f). Similar to ASB2 $\alpha$, ASB2 $\beta$ can interact with elongin $B$, elongin $C$, Cullin5 and Rbx2 whereas an ASB2 $\beta$ BC-box mutant (ASB2 $\beta$ LA) did not (Figure 4f). To determine whether the ASB2 $\beta$ /elongin $\mathrm{BC} / \mathrm{Cullin} 5 / \mathrm{Rb} 2 \mathrm{2}$ complex possesses ubiquitin ligase activity, we immunoaffinity-purified and assayed the complex for its ability to activate formation of polyubiquitin chains by the E2 ubiquitin-conjugating enzyme $\mathrm{UbcH} 5 \mathrm{a}$ in the presence of ATP, E1 ubiquitin-activating enzyme Uba1 and ubiquitin. As shown in Figure $4 \mathrm{~g}$, the ASB2 $\beta$ complex stimulated formation of ubiquitin conjugates by E2. In contrast, anti-Flag immunoprecipitation from lysates of insect cells not expressing ASB2 $\beta$ did not support formation of polyubiquitin conjugates (Figure $4 \mathrm{~g}$ ). Furthermore, the ASB2 $\beta$ BC-box mutant that cannot assemble with elongin $\mathrm{B}$, elongin $\mathrm{C}$, Cullin5 and $\mathrm{Rbx} 2$ did not stimulate the polyubiquitylation activity of $\mathrm{UbcH} 5 \mathrm{a}$ (Figure $4 \mathrm{~g}$ ). Among the proteins that were polyubiquitylated in this in vitro ubiquitylation reaction, ASB2 $\beta$ and Cullin5 were found to be polyubiquitylated (Figure $4 \mathrm{~h}$ ). Altogether, our results indicated that ASB2 $\beta$ can assemble with elongin $\mathrm{B}$, elongin $\mathrm{C}$, Cullin5 and Rbx2 to reconstitute an active E3 ubiquitin ligase complex.

\begin{abstract}
ASB2 $\beta$ triggers ubiquitylation and proteasome degradation of FLNb. We previously reported that ASB2 $\alpha$ targets FLNa and FLNb to proteasome degradation. ${ }^{6}$ We therefore assessed the expression of the ubiquitously expressed FLNa and FLNb as well as the muscle-specific FLNc during differentiation of $\mathrm{C} 2 \mathrm{C} 12$ cells. Interestingly, ASB2 $\beta$ upregulation correlated with loss of FLNb (Figure 5a). In contrast, expression of FLNa was not regulated and expression of FLNc was induced (Figure 5a). As shown in Figure $5 b$, accelerated degradation of FLNb was observed in differentiating $\mathrm{C} 2 \mathrm{C} 12$ cells compared to proliferating cells. Furthermore, loss of FLNb was mediated by the proteasome as treatment of differentiating $\mathrm{C} 2 \mathrm{C} 12$ cells with the proteasome inhibitor MG132 reduced FLNb degradation (Figure 5c). Altogether, these suggest that FLNb may be a substrate of ASB2 $\beta$. To determine whether ASB2 $\beta$ can promote FLNb ubiquitylation, we performed in vitro substrate ubiquitylation assays using purified GFP-tagged FLNb. When FLNb-GFP was used as a substrate, ubiquitylation of FLNb by $\mathrm{UbcH} 5 \mathrm{a}$ in the presence of the ASB2 $\beta /$ elongin $\mathrm{BC} / \mathrm{Cullin} 5 / \mathrm{Rbx} 2$ or the ASB2 $\alpha$ /elongin BC/Cullin5/Rbx2
\end{abstract}

complexes but not in the presence of ASB2 E3 ligase defective mutants (ASB2 $\beta$ LA or ASB2 $\alpha$ LA) was observed (Figure 5d). To confirm these results, we co-transfected NIH3T3 cells with vectors expressing GFP, GFP-ASB2 $\beta$, GFP-ASB $2 \beta$ LA, GFP-ASB $2 \alpha$ or GFP-ASB $2 \alpha$ LA together with a FLNb-GFP or a FLNa-GFP expression vector. At $24 \mathrm{~h}$ after transfection, western blotting revealed that GFP-ASB2 $\beta$ expression resulted in a loss of FLNb-GFP (Figure $5 e$ ) but not of FLNa-GFP (Figure 5f). However, loss of both FLNaGFP and FLNb-GFP was observed in cells transfected with a GFP-ASB2 $\alpha$ expression vector as previously reported. ${ }^{6}$ Furthermore, FLNb-GFP levels were not altered in cells expressing GFP-ASB2 $\beta$ LA (Figure 5e). As expected, proteasome inhibitors blocked FLNb-GFP degradation induced by GFP-ASB2 $\beta$ (Figure 5e). To determine whether ASB2 $\beta$ induces degradation of endogenous FLNb, we transfected $\mathrm{C} 2 \mathrm{C} 12$ myoblasts with vectors expressing GFP-ASB2 $\beta$ or GFP-ASB2 $\beta$ LA. At $20 \mathrm{~h}$ after transfection, FLNb could not be detected in cells expressing GFP. ASB2 $\beta$ wt whereas FLNb expression was unaffected by GFP-ASB2 $\beta$ LA (Figure $5 \mathrm{~g}$ ). To extend and further validate the finding that $A S B 2 \beta$ induced FLNb degradation during myogenic differentiation, we investigated ASB2 $\beta$ and FLNb expression during differentiation of human primary myoblasts. Differentiation was confirmed by their morphological changes (Figure 6a) and the expression of myogenin, $\mathrm{MHC}$ and troponin $\mathrm{T}$ (Figure $6 \mathrm{~b}$ ) after switching cells from GM to DM. In these cells, ASB2 $\beta$ upregulation correlated with decrease of FLNb (Figure 6b). Altogether, our results indicate that $\mathrm{ASB} 2 \beta$ ubiquitin ligase activity drives ubiquitin-mediated proteasomal degradation of FLNb.

Inhibition of ASB2 $\beta$ expression blocks myoblast fusion and myotube formation. To determine whether ASB2 $\beta$ is required for myoblast differentiation, we generated ASB2 $\beta$ knockdown stable cell populations by transfection of vectors encoding short-hairpin RNAs (shRNAs) directed against ASB2 $\beta$. Knockdown of ASB2 $\beta$ expression in $\mathrm{C} 2 \mathrm{C} 12$ cells cultured in DM was demonstrated by northern blot (data not shown) and western blot (Figure 7a and b) analyses. In these cells, FLNb degradation was delayed (Figure 7a and b). Knockdown of ASB2 $\beta$ expression delayed myotube formation as evaluated by morphological observations (Figure 7c) and confirmed by the reduction in the level of both $\mathrm{MHC}$ and troponin $\mathrm{T}$ expression (Figure 7f).

Figure 5 ASB2 $\beta$ induced ubiquitin-mediated FLNb degradation. (a) Expression of FLNa, FLNb and FLNc in C2C12 cells induced to differentiate. C2C12 cells were cultured in growth medium (GM) and shifted to differentiation medium (DM) for 6 days. Expression of FLNa, FLNb, FLNc and Erk2 was analyzed by western blot using $20 \mu \mathrm{g}$ aliquots of whole-cell extracts. (b) Quantification of FLNb turnover following cycloheximide (CHX) treatment. C2C12 cells were cultured in GM or shifted to DM for $18 \mathrm{~h}$ and subsequently treated with $\mathrm{CHX}$ to block protein synthesis for various times (1.5, 3 and $6 \mathrm{~h})$. (c) FLNb degradation in differentiating C2C12 cells is dependent on the proteasome. $\mathrm{C} 2 \mathrm{C} 12$ cells were cultured in DM for $16 \mathrm{~h}$ and subsequently treated with MG132 or DMSO for $8 \mathrm{~h}$. In b and c, $10 \mu \mathrm{g}$ aliquots of each whole-cell extract were immunoblotted with antibodies to FLNb and Erk2 and quantification of FLNb level relative to Erk2 by densitometric scanning of three independent experiments is shown. (d) ASB2 $\beta$ induces polyubiquitylation of FLNb. Recombinant ASB2/elongin BC/Cullin5/Rbx2 complexes were purified as in Figure 3f. All samples contained purified Uba1, UbcH5a, ubiquitin, APP-BP1/Uba3, Ubc12 and NEDD8. Purified FLNb-GFP was also provided as indicated and subjected to ubiquitylation. Aliquots of the reaction mixture were analyzed by western blotting using anti-GFP (upper panel) and antibodies to polyubiquitylated proteins (lower panel). (e) ASB2 $\beta$-induced FLNb degradation is dependent on ASB2 ubiquitin ligase activity and the proteasome. NIH3T3 cells were mock-transfected or transfected for $24 \mathrm{~h}$ with FLNb-GFP together with GFP (-), or GFP-ASB2 $\alpha$ wt, GFP-ASB2 $\alpha$ LA, GFP-ASB2 $\beta$ wt, GFP-ASB2 $\beta$ LA expression vectors in the absence or presence of $1 \mu \mathrm{M}$ MG132 for $18 \mathrm{~h}$, as indicated. (f) ASB2 $\beta$ does not induce FLNa degradation. NIH3T3 cells were mock-transfected or transfected for $24 \mathrm{~h}$ with FLNa-GFP together with GFP (-), or GFP-ASB2 expression vectors, as indicated. In e and $\mathrm{f}$, $20 \mu \mathrm{g}$ aliquots of whole-cell extracts were immunoblotted with antibodies to GFP. (g) ASB2 $\beta$ induced degradation of endogenous FLNb. C2C12 myoblasts were transfected with GFP-ASB2 $\beta$ wt or GFP-ASB2 $\beta$ LA expression vectors, plated on fibronectin-coated coverslips $5 \mathrm{~h}$ after transfection, fixed $15 \mathrm{~h}$ later and analyzed using an antibody directed against FLNb. Scale bar, $10 \mu \mathrm{m}$ 
Furthermore, quantification of the fusion index demonstrated that $A S B 2 \beta$ is required for myotube formation (Figure $7 d$ and $e$ ). Conversely, the cell population transfected with an empty vector formed myotubes and expressed markers of muscle differentiation upon a shift to DM as expected (Figure 7c-f). To demonstrate the involvement of FLNb degradation in
ASB2 $\beta$-mediated effects on cell differentiation, we have investigated whether FLNb knockdown in ASB2 $\beta$ knockdown $\mathrm{C} 2 \mathrm{C} 12$ cells can rescue the differentiation defects of these cells. Therefore, we have generated stable FLNb knockdown in ASB2 $\beta$ knockdown $\mathrm{C} 2 \mathrm{C} 12$ cells. In these cells, FLNb expression was reduced to $50 \%$ compared to ASB2 $\beta$ a

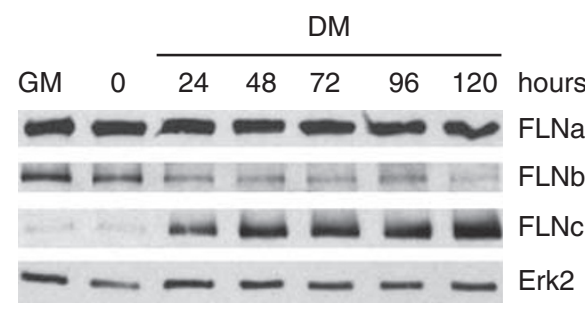

C

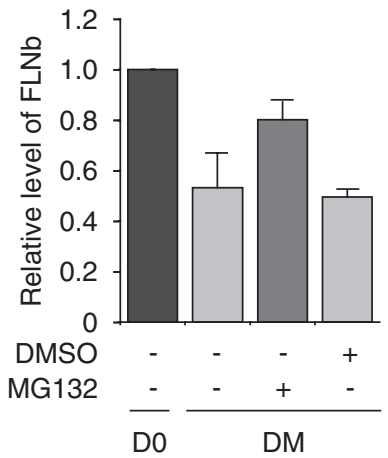

e

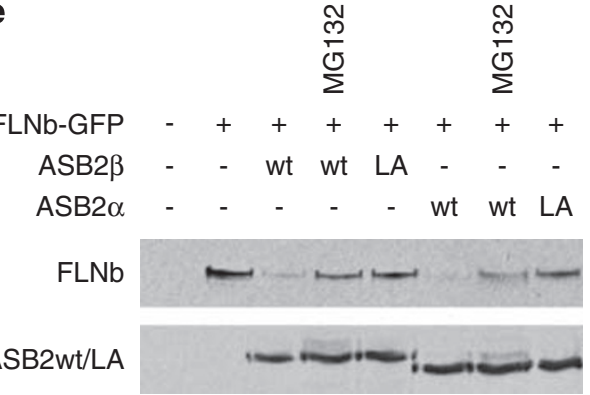

d

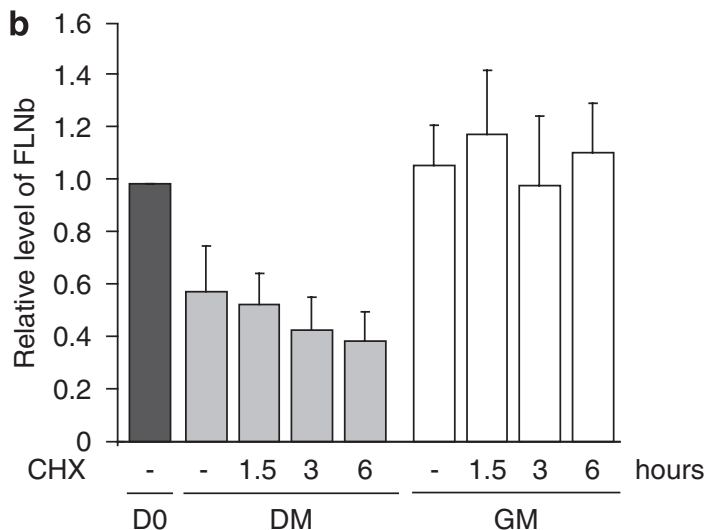

ASB2 $\beta$ - wt wt LA - - -

ASB2 $\alpha$ - - - - wt wt LA

$\mathrm{EIBC} / \mathrm{Cul} 5 / \mathrm{Rbx} 2-++++++$

$\mathrm{FLNb}+-++-++$

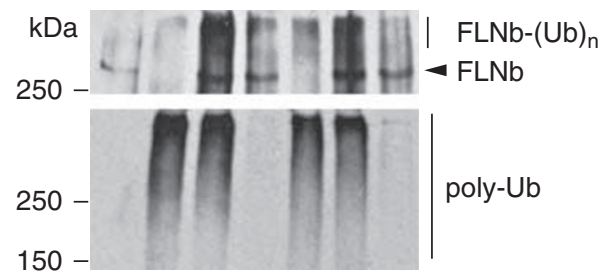

\section{f}

FLNa-GFP -+++++

ASB2 $\beta$ - - - - wt LA

ASB2 $\alpha$ - - wt LA - -

$\mathrm{FLNa}$

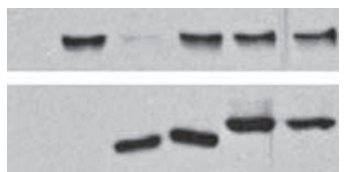

ASB2wt/LA

g

FLNb

Overlay
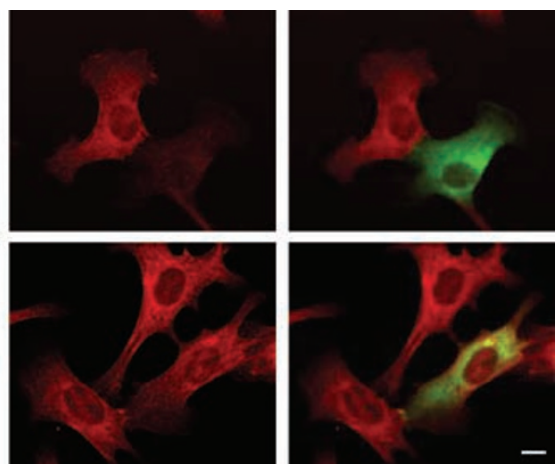
knockdown cells expressing constructs that generate an shRNA targeting luciferase as controls (Figure 7g). The low level of FLNc present in undifferentiated $\mathrm{C} 2 \mathrm{C} 12$ cells was not increased in ASB2 $\beta$ knockdown cells, indicating that there is no functional compensation between FLNb and FLNc in these cells (Figure 7g). When cultured in DM, ASB2 $\beta / F L N b$ knockdown cells differentiate more rapidly than ASB2 $\beta$ knockdown cells transfected with a vector expressing the control shRNA as demonstrated by the expression of differentiation markers (Figure 7h). Altogether, our results indicated that ASB2 $\beta$ is required for the differentiation of C2C12 myoblasts into myotubes and regulates cell differentiation through FLNb degradation.

\section{Discussion}

The ASB2 gene was originally identified as a retinoic acid-inducible gene whose expression recapitulates early differentiation events critical to induced differentiation of myeloid leukemia cells. ${ }^{3}$ EST database searches identified a

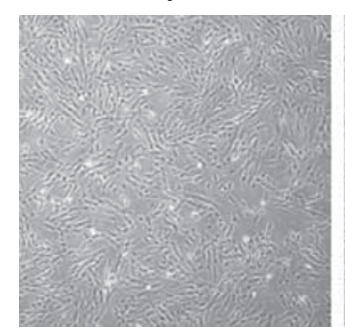

day 3

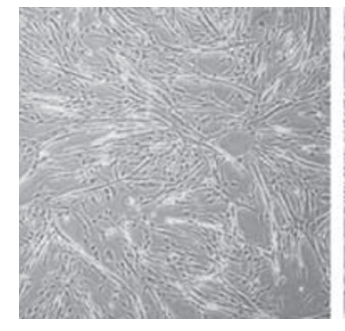

b
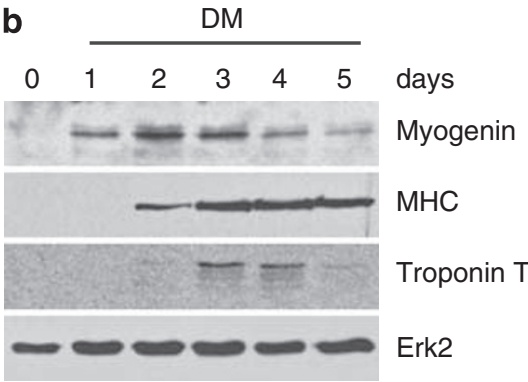

day 1

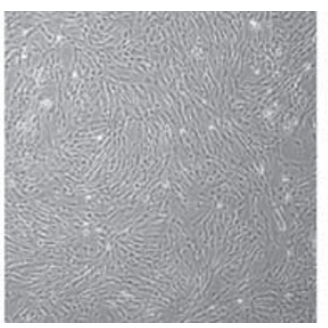

day 4

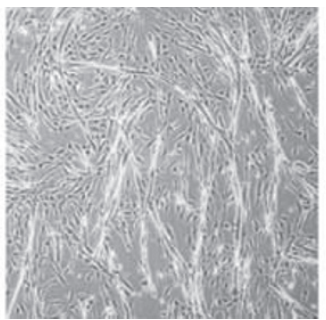

day 2

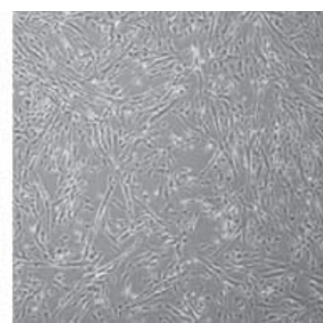

day 5

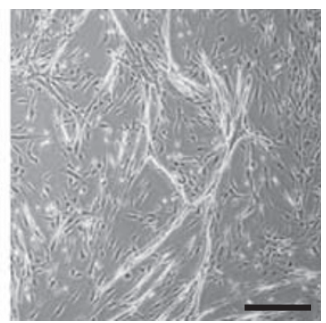

c

DM

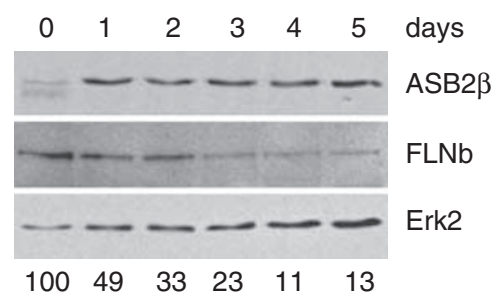

Figure 6 Downregulation of FLNb in primary myoblasts induced to differentiate correlates with ASB2 $\beta$ induction. Human myoblasts were cultured in growth medium (GM) and shifted to differentiation medium (DM) for 5 days. (a) Morphological changes of primary cells for assessment of alignment, elongation and fusion were observed under a phase-contrast microscope. Scale bar, $500 \mu \mathrm{m}$. (b) Expression of myogenin, MHC, troponin T and Erk2 during differentiation of primary cells. (c) Expression of ASB2 $\beta$, FLNb and Erk2 during differentiation of primary cells. The numbers under the blot represent the percentages of FLNb relatively to day 0 , calculated after normalization to Erk2. In $\mathbf{b}$ and $\mathbf{c}, 5 \mu \mathrm{g}$ aliquots of each whole-cell extract were analyzed by western blot using indicated antibodies

Figure 7 ASB2 $\beta$ knockdown delayed differentiation of $\mathrm{C} 2 \mathrm{C} 12$ myoblasts. (a-f) Stable $\mathrm{C} 2 \mathrm{C} 12$ cell populations expressing shRNAs directed against ASB2 $\beta$ (ASB2 $\beta$ KD1 and ASB2 $\beta$ KD2) or transfected with the empty vector (ctrl) were shifted to DM for 5 days. (a) Aliquots $(20 \mu \mathrm{g})$ of urea soluble fractions were analyzed by western blot using 2PNAB1 serum. Aliquots $(5 \mu \mathrm{g})$ of whole-cell extracts were analyzed by western blot using anti-FLNb (N-16) and anti-Erk2 antibodies. (b) Expression of FLNb relative to Erk2 based on densitometric scanning. Results are mean \pm standard deviation of three independent experiments. (c) Images of differentiating C2C12 cell populations (1, 3 and 5 days after initiation of differentiation) observed with a phase-contrast microscope are shown for each stable cell populations. Scale bar, $100 \mu \mathrm{m}$. (d) Fluorescence images of $\mathrm{C} 2 \mathrm{C} 12$ cell populations, 5 days after induction of differentiation. Troponin T (green) and nuclei (blue) were stained to identify troponin T positive cells and facilitate myotube identification. Scale bar, $20 \mu \mathrm{m}$. (e) Histograms represent the fusion index calculated for $\mathrm{C} 2 \mathrm{C} 12$ cell populations at day 3 and 5 . Results are mean \pm standard deviation from two independent experiments, where at least 200 nuclei per experiment were counted. (f) Expression of myosin heavy chain (MHC) and troponin T during differentiation of C2C12 cell populations. Aliquots $(10 \mu \mathrm{g})$ of each whole-cell extract were analyzed by western blot, using anti-MHC and anti-troponin T antibodies. (g) FLNb knockdown in ASB2 $\beta$ knockdown C2C12 cells. ASB2 $\beta$ KD2 cells were stably transfected with constructs that generate shRNAs targeting ASB2 $\beta$ (ASB2 $\beta / F L N b$ KD) or luciferase (ASB2 $\beta$ l Luc KD). Aliquots $(10 \mu \mathrm{g})$ of each whole-cell extract were immunoblotted with antibodies to FLNb, FLNc and Erk2. (h) Expression of MHC and troponin T during differentiation of ASB2 $\beta$ and FLNb double knockdown C2C12 cells. ASB2 $\beta / F L N b ~ K D$ and ASB2 $\beta / L u c ~ K D$ cells were shifted to DM for 6 days. Aliquots (10 $\mu \mathrm{g})$ of each whole-cell extract were analyzed by western blotting using indicated antibodies 
two different ASB2 protein isoforms, a hematopoietic type and a muscle type, that we named ASB2 $\alpha$ and ASB2 $\beta$, respectively. Our results show that ASB2 $\beta$ mRNAs are expressed in muscle cells whereas ASB $2 \alpha \mathrm{mRNAs}$ are mainly expressed in hematopoietic cells. Whether the tissue-specific control of ASB2 transcription is achieved through two different

a $\begin{array}{llllllllllllllll}0 & 1 & 2 & 3 & 5 & 0 & 1 & 2 & 3 & 5 & 0 & 1 & 2 & 3 & 5 & \text { days }\end{array}$ ASB2 $\beta$ ant- $=$

$\mathrm{FLNb}$

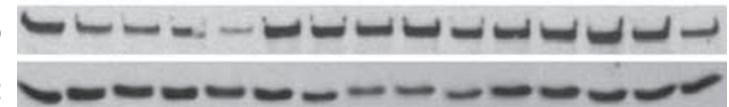

Erk2
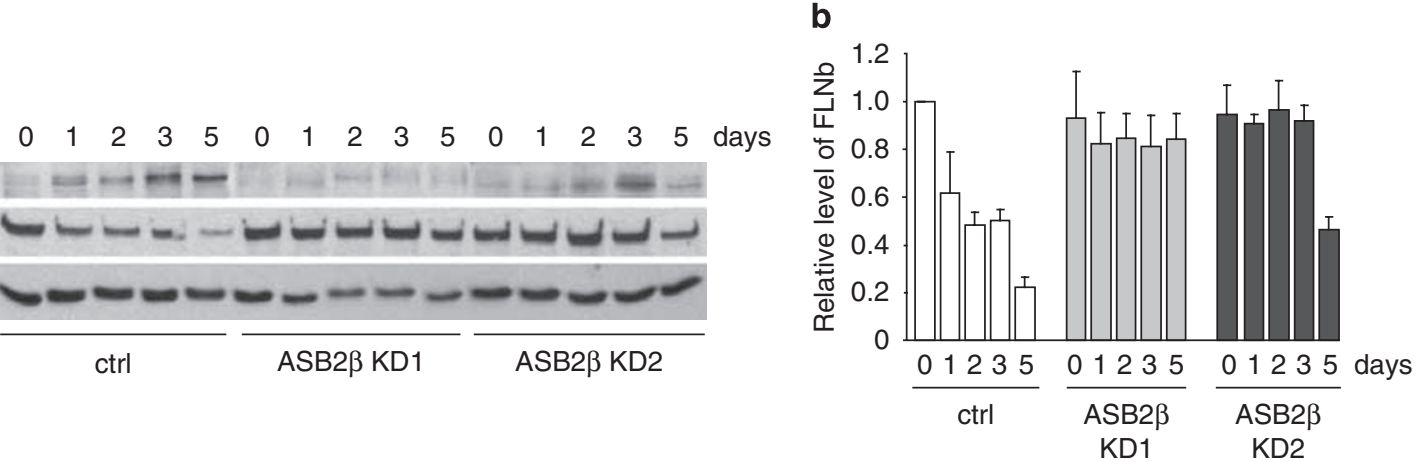

C

day 0

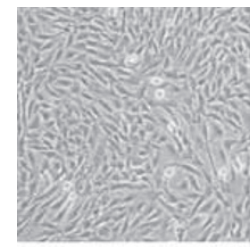

ctrl

ASB2 $\beta$

KD1

ASB2 $\beta$

KD2 day 1
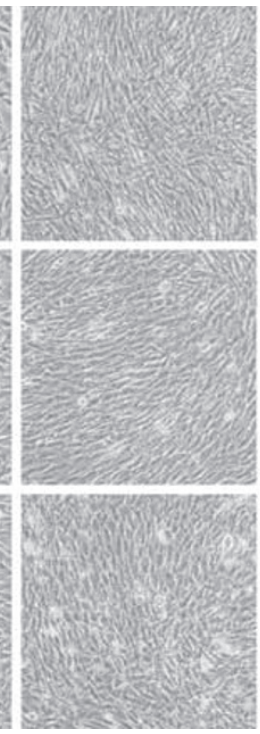

e

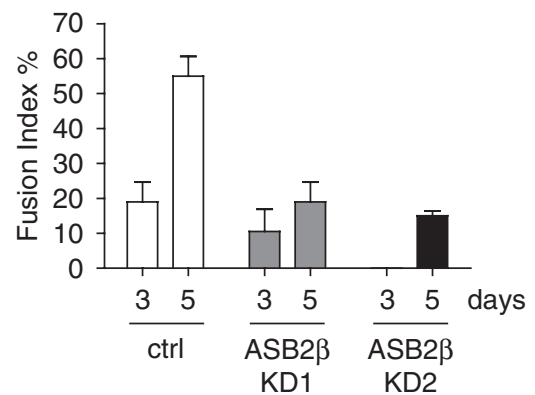

g

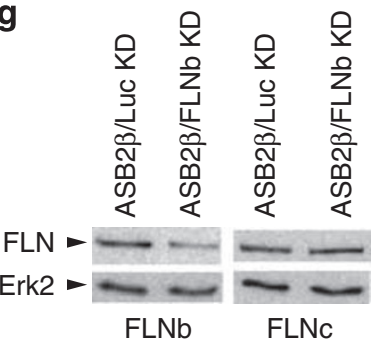

day 3
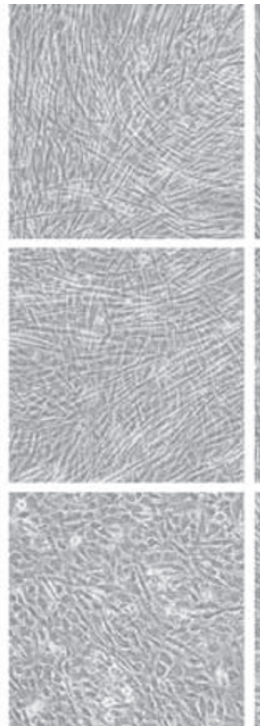

f

0123350012335001235 days

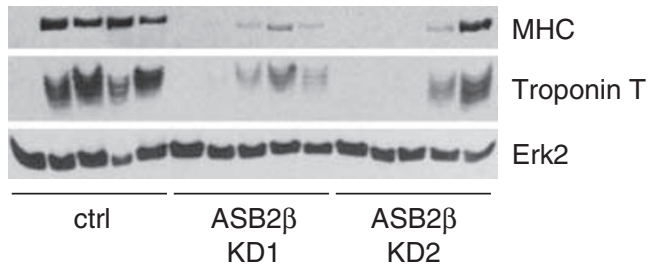

h 01234560123456 days

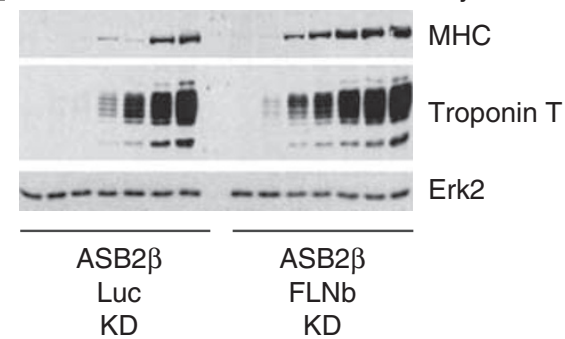

day 5
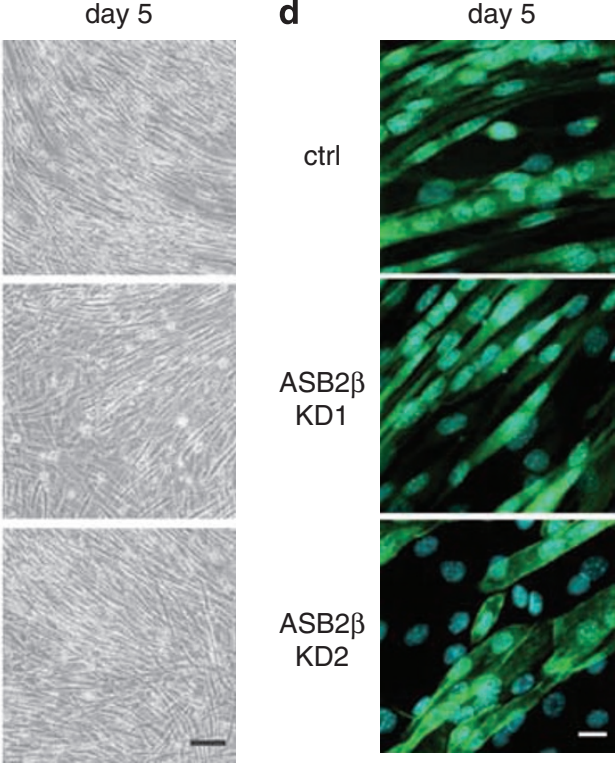
promoters resulting in the synthesis of two cell-specific ASB2 isoforms is the subject of ongoing experiments. We further demonstrate that ASB2 $\beta$-specific antibodies recognize a $70 \mathrm{kDa}$ protein in differentiated muscle cells. The two ASB2 isoforms differ in their $\mathrm{N}$-terminal region but share ankyrin repeats and an SOCS box. As demonstrated for ASB2 $\alpha$, $\mathrm{ASB} 2 \beta$, by interacting with the elongin $\mathrm{BC}$ complex, can assemble with Cullin5 and Rbx2 to form a bona fide multimeric really interesting new gene (RING)-type E3 ubiquitin ligase complex that stimulates polyubiquitylation by the E2 ubiquitin-conjugating enzyme UbcH5a. The ASB2 $\beta$ isoform harbors a UIM at its $\mathrm{N}$ terminus. The UIM was initially identified in the proteasomal S5a subunit as involved in recognition of ubiquitylated substrates. ${ }^{25}$ UIMs form a single $\alpha$-helix that binds polyubiquitin chains as well as monoubiquitin and promotes ubiquitylation of proteins that contain them. ${ }^{26}$ Recently, the UIM motif of Met4 was shown to protect polyubiquitylated Met4 from proteolysis by the proteasome. ${ }^{27}$ Whether ASB2 UIM is involved in the regulation of $A S B 2$ stability and/or activity remains to be determined.

Although it is known that ASB proteins are implicated in diverse biological functions such as hematopoiesis, the substrates that are targeted for polyubiquitylation by ASB proteins are largely undefined. Although ASB2 $\alpha$ induces proteasomal degradation of both FLNa and FLNb (Heuze et al. ${ }^{6}$; this report), we showed here that ASB2 $\beta$ induces FLNb ubiquitylation and subsequently FLNb degradation, indicating that ASB2 $\beta$ is specific for FLNb over FLNa. Furthermore, knockdown of endogenous ASB2 $\beta$ in $\mathrm{C} 2 \mathrm{C} 12$ cells delays myogenic differentiation and FLNb degradation. These results are in line with the previously reported FLNb downregulation during $\mathrm{C} 2 \mathrm{C} 12$ myoblast differentiation. ${ }^{28}$ Because loss of $\mathrm{FLNb}$ is markedly delayed in ASB2 $\beta$ KD2 cells, we cannot exclude that a threshold of ASB2 $\beta$ is necessary to target FLNb to proteasomal degradation. Furthermore, we cannot exclude the possibility that other ASB2 $\beta$ targets are also important for differentiation of $\mathrm{C} 2 \mathrm{C} 12$ cells.

Cell migration is a crucial step in skeletal muscle development during which myogenic progenitors migrate from the somites to the limb musculature. To differentiate and fuse to form syncytial skeletal muscle fibers, myoblasts must become less motile and establish cell-cell and cell-extracellular matrix contacts leading to cytoskeletal rearrangements. ${ }^{29}$ In this regard, it is noteworthy that $A S B 2$ is expressed in the myotome of the somites and in the limb during chick embryogenesis. This together with the fact that ASB2 $\beta$ can regulate the degradation of $\mathrm{FLNb}$, a protein involved in actin remodeling, suggest that ASB $\beta$ can contribute to cytoskeletal reorganization during myogenesis. The appearance of ASB2 $\beta$ marks a very early event in differentiation of $\mathrm{C} 2 \mathrm{C} 12$ cells; ASB2 $\beta$ was upregulated at the same time as myogenin, the earliest known marker of myoblasts committed to the differentiation pathway expressed before the establishment of the postmitotic state. ${ }^{30}$ Inactivation of ASB2 $\beta$ by shRNAs interfered with the normal induction of muscle-specific proteins in $\mathrm{C} 2 \mathrm{C} 12$ cells and delayed myotube formation. This suggests that $A S B 2 \beta$ is important for myogenic differentiation. Furthermore, a partial knockdown of FLNb in ASB2 $\beta$ knockdown $\mathrm{C} 2 \mathrm{C} 12$ cells accelerated the induction of differentiation markers, demonstrating that the cell differentiation defect of ASB2 knockdown cells is due, at least in part, to its effect on FLNb degradation.

Interestingly, ASB2 $\beta$ induction and subsequent FLNb downregulation correlates with the switch of the $\beta 1 \mathrm{~A}$ to the $\beta 1 \mathrm{D}$ splice variant of the integrin $\beta 1$ subunit associated with the commitment to differentiation of $\mathrm{C} 2 \mathrm{C} 12$ myoblasts. A critical function of integrins during myogenesis has been proposed since antibody ligation of $\beta 1$ integrins perturbed myotube formation in vitro ${ }^{31}$ and inactivation of the mouse $\beta 1$ integrin gene in developing myoblasts inhibited myoblast fusion and sarcomere assembly. ${ }^{32}$ Previous reports have indicated that FLNb binds strongly to $\beta 1 \mathrm{~A}$ integrin but poorly to $\beta 1 \mathrm{D}$ whereas talin binds strongly to $\beta 1 \mathrm{D}$ and with intermediate affinity to $\beta 1 \mathrm{~A} .{ }^{28,33}$ Thus, differential binding of FLNb or talin to $\beta 1 \mathrm{~A}$ integrin may modulate integrin-dependent functions such as cytoskeleton remodeling and signaling. It is therefore tempting to speculate that $\mathrm{ASB} 2 \beta$ may impact integrindependent functions. Our results provide a mechanism through which expression of FLNb and integrins are coordinately regulated, allowing myogenic differentiation. Alternatively, because FLNs act as scaffolds for signaling molecules involved in actin remodeling and/or transcriptional regulation, ASB2 $\beta$ may regulate pathways downstream of FLNb that have to be activated during muscle differentiation. Which signal transduction pathways are regulated is the subject of ongoing investigation.

Expression of $A S B 2$ in axial and limb skeletal muscles during chick embryogenesis is consistent with ASB2 $\beta$ expression in myotubes. ASB2 $\beta$ is expressed in adult muscles suggesting that $A S B 2 \beta$ may also have a function in muscle remodeling. Interestingly, $\mathrm{ASB} 2 \beta$ is upregulated during mouse embryonic development at 17 d.p.c., a period associated with synaptic connections. Hence, it will be important to determine whether ASB2 $\beta$ expression correlates with muscle innervation. Furthermore, in a recent work aimed to the identification of transcripts with a circadian pattern of expression in adult skeletal muscle, atrogin-1, MURF1 as well as $A S B 2$ were found to be circadian genes, ${ }^{34}$ suggesting that these E3 ubiquitin ligases have a function in maintaining cellular homeostasis in skeletal muscle cells. An interesting possibility is that ASB2 $\beta$ regulates independent mechanisms in myoblasts and skeletal myotubes. In this regard, it will be important to identify ASB2 $\beta$ substrate(s) in fully differentiated muscle cells. Future studies will also be necessary to further our understanding of FLNb function in myoblasts.

\section{Materials and Methods}

Cell lines, culture conditions, induction and differentiation. The mouse myoblasts of the $\mathrm{C} 2 \mathrm{C} 12$ cell line were grown in Dulbecco's modified Eagle's medium (DMEM) containing $4.5 \mathrm{~g} / \mathrm{l}$ glucose, $10 \%$ fetal bovine serum (PAA Laboratories), $1 \%$ sodium pyruvate, $1 \%$ nonessential amino acids and penicillinstreptomycin (Invitrogen). For differentiation studies, $\mathrm{C} 2 \mathrm{C} 12$ cells were plated at 7500 cells per $\mathrm{cm}^{2}$, grown to $80 \%$ confluence in 2 days and then cultured in differentiation medium containing DMEM supplemented with $2 \%$ horse serum (PAA Laboratories). Differentiation medium was changed every $48 \mathrm{~h}$. The fusion index, that is, the number of nuclei in troponin T positive multinucleated myotubes divided by the total number of nuclei, calculated for $\mathrm{C} 2 \mathrm{C} 12$ parental cells at day 6 was $65 \%$. Human primary myoblasts isolated from a quadriceps muscle biopsy of a newborn infant as described ${ }^{35}$ were obtained from V Mouly (Institut de Myologie, Paris, France). Human myoblasts were grown in F10 medium (Invitrogen) supplemented 
with $20 \%$ fetal bovine serum and penicillin-streptomycin. For differentiation studies, human myoblasts were plated at 3500 cells per $\mathrm{cm}^{2}$, grown to $80 \%$ confluence in 6 days and then cultured in differentiation medium containing DMEM supplemented with $10 \mu \mathrm{g} / \mathrm{ml}$ insulin (Sigma-Aldrich) and $100 \mu \mathrm{g} / \mathrm{ml}$ transferin (Sigma-Aldrich). Differentiation medium was changed every $24 \mathrm{~h}$. HeLa cells were grown on Petri dishes in DMEM containing $4.5 \mathrm{~g} / \mathrm{l}$ glucose, $10 \%$ fetal bovine serum (PAA Laboratories), glutamax, pyruvate and penicillin-streptomycin (Invitrogen). NIH3T3 cells were grown in DMEM containing $4.5 \mathrm{~g} / \mathrm{l}$ glucose (Invitrogen), $1 \%$ sodium pyruvate, $10 \%$ newborn calf serum (PAA Laboratories) and penicillin-streptomycin. NB4 cells were used as described. ${ }^{3}$ Cells were maintained in a $5 \% \mathrm{CO}_{2}$ incubator at $37^{\circ} \mathrm{C}$. For proteasome inhibition, $\mathrm{NIH} 3 \mathrm{~T} 3$ and $\mathrm{C} 2 \mathrm{C} 12$ cells were incubated with 1 and $5 \mu \mathrm{M}$ MG132 (Euromedex), respectively. To inhibit de novo protein synthesis, we treated $\mathrm{C} 2 \mathrm{C} 12$ cells with $5 \mu \mathrm{g} / \mathrm{ml}$ cycloheximide (Sigma-Aldrich).

Plasmid constructs. The pCMVSport6-mASB2 $\beta$ vector was obtained from RZPD (Deutsches Ressourcenzentrum für Genomforschung $\mathrm{GmbH}$ ). The mASB2 $\beta$ open-reading frame was subcloned into a pBacPAK9 (Clontech)-derived vector to direct the expression of $\mathrm{mASB} 2 \beta$ fused to the Flag epitope at its $\mathrm{N}$ terminus (pBacPAK9FN-mASB2 $\beta$ ), into a pCMV-derived vector to direct the expression of mASB2 $\beta$ tagged with two Flag epitopes at its $N$ terminus (pCMV-Flag2-mASB2 $\beta$ ) and into the pEGFP-C3 expression vector (Clontech). Mutation L595A was introduced into $\mathrm{mASB} 2 \beta$ using the QuikChange site-directed mutagenesis kit (Stratagene) and the mutated oligonucleotide sequence, as indicated in boldface $5^{\prime}$-CTCCGAGACCTGCGGCTCACCTCTGCCG-3'. The pcDNA3-cASB2 plasmid was obtained from the University of Delaware and contains the $3^{\prime}$ end of chicken ASB2 cDNA (accession number Al982288). The hASB2 $\alpha$ open-reading frame ${ }^{3,4}$ was subcloned into the pCMV-Flag2 generating the pCMV-Flag2-ASB2 $\alpha$ vector. The pcDNA3-FLNa-GFP, ${ }^{36}$ pCl-puro-FLNb-GFP ${ }^{28}$ and pEGFP-C3-ASB2 $\alpha^{6}$ expression constructs have been used previously.

Specific silencing of mASB2 $\beta$ was achieved by using an shRNA-expressing vector. Nucleotides 96-114 (sh\#1) and 1370-1388 (sh\#2) of the mASB2 $\beta$ coding sequence were chosen as target for shRNA. The shRNA sequences were used to construct 60-mer shRNA oligonucleotides, which were then synthesized (MWG) and ligated into the pSUPER.neo.gfp expression vector (Oligoengine) under the contro of the $\mathrm{H} 1$ promoter. The following oligonucleotides were used (underlined, sense and antisense sequences; boldface, restriction enzyme sites; lightface italics, pollII termination signals; boldface italics, loop with linker): sh\#1: $5^{\prime}$-GATCCCCGAGTC ATAACGTCTTATAGTTCAAGAGACTATAAGAACGTTATGACTC TTITTGGAAA-3'; sh\#2: 5'-GATCCCCCGCCGATGCTAACAAAGCCTTCAAGAGAGGCTTTGTTAG CATCGGCGTTTTTGGAAA-3'. All constructs were verified by DNA sequencing.

Northern blotting. Total RNA was isolated from mouse tissues following the method of Chomczynski and Sacchi ${ }^{37}$ and from $\mathrm{C} 2 \mathrm{C} 12$ cells using a nucleospin RNA II kit (MACHEREY-NAGEL). Hybridization was as described. ${ }^{38}$ The ASB2 probe corresponded to the mouse ASB2 $\beta$ open-reading frame. The human RNA Master blot and the mouse embryo MTN blot were obtained from Clontech. PolyA + RNA samples on Master blot have been normalized to the mRNA levels of eight different 'housekeeping' genes. The $\beta$-actin probe was from Clontech and the Arbp probe was previously described as $36 \mathrm{~B} 4$ probe.

Quantitative RT-PCR. Total mRNAs from human skeletal muscle, heart and smooth muscle were from Clontech. Total mRNA was extracted and purified from NB4 acute promyelocytic leukemia cells treated for 2 days with $10^{-6} \mathrm{M}$ all-trans retinoic acid as described. ${ }^{3}$ cDNA was synthesized using superscript III first-strand synthesis kit as recommended by the manufacturer (Invitrogen). cDNA synthesis experiments were repeated three times. Real-time PCR was carried out with the 7300 real-time PCR system using the SYBR Green PCR master mix (Applied Biosystems) according to the manufacturer's instructions. The specificity of the PCR primers was confirmed by melting curve analyses. Primers for detection of human ASB2 mRNAs were designed based on chromosome 14 sequence according to the requirements for real-time RT-PCR using the Perl Primer software. Oligonucleotide primer sequences corresponding to distinct exons were as follows: forward $5^{\prime}$-AT TCCTGCCTGAAGCC- $3^{\prime}$ and reverse $5^{\prime}$-TGCAGTGGACCTGGA- $3^{\prime}$ for ASB2 $\alpha$ forward $5^{\prime}$-GAATTGTACCCTTGTTTCAGAG- $3^{\prime}$ and reverse $5^{\prime}$-CTCCAGAA CAGACACCC- $3^{\prime}$ for ASB2 $\beta$; forward $5^{\prime}$-GCCCAGAGTGGACAGTTGGA- $3^{\prime}$ and reverse $5^{\prime}$-TGGCCTGCGTGTTGATGT- $3^{\prime}$ common to both ASB2 isoforms. Efficiency of amplification was determined using the standard curves method. Fold changes were quantified as $2^{-(\mathrm{Ct} \text { isoform-Ct common) }}$
In situ hybridization. In situ hybridization to whole-mount embryos and to tissue sections was performed at various developmental stages, ranging from E2 to E10 as previously described. ${ }^{39}$ The fragment corresponding to part of the coding sequence of chicken ASB2 was isolated from pcDNA3-CASB2 and used to generate a single-stranded antisense digoxigenin-labeled RNA probe.

Transfections. Exponentially growing HeLa and $\mathrm{NIH} 3 \mathrm{~T} 3$ cells were transfected using the Jet PEI reagent (Polyplus transfection) as recommended by the manufacturer. For transient expression, $\mathrm{C} 2 \mathrm{C} 12$ cells were transfected using Lipofectamine as per manufacturer's instructions. To establish stable transfectants, we transfected $\mathrm{C} 2 \mathrm{C} 12$ cells using the nucleofector $\mathrm{V}$ solution and the $\mathrm{B} 32$ program, as recommended by the manufacturer (Amaxa). ASB2 $\beta$ knockdown was obtained transfecting $\mathrm{C}_{2} \mathrm{C} 12$ cells with shRNA against mouse ASB2 $\beta$. Cells were then cultured for $48 \mathrm{~h}$ before selection with $0.5 \mathrm{mg} / \mathrm{ml} \mathrm{G} 418$ (Invitrogen). FLNb knockdown was obtained transfecting $\mathrm{C} 2 \mathrm{C} 12$ cells that have been previously transfected with sh\#2 directed against ASB2 $\beta$ with an shRNA against mouse FLNb in pGIPZ vector (Open Biosystems). A vector expressing an shRNA in pGIPZ vector targeted to luciferase (Open Biosystems) was used as a control. After 2 days, we selected the transfected cells using $1 \mu \mathrm{g} / \mathrm{ml}$ puromycin together with $0.5 \mathrm{mg} / \mathrm{ml}$ G418.

Whole-cell extracts. $\mathrm{C} 2 \mathrm{C} 12$ cells were washed twice in PBS and resuspended in whole-cell extract buffer containing $50 \mathrm{mM}$ Tris- $\mathrm{HCl}(\mathrm{pH} 7.9)$, $150 \mathrm{mM} \mathrm{NaCl}, 1 \mathrm{mM}$ EDTA, $0.1 \%$ NP-40, 10\% glycerol, $1 \mathrm{mM}$ dithiothreitol, $1 \mathrm{mM}$ $\mathrm{Na}_{3} \mathrm{VO}_{4}, 50 \mathrm{mM} \mathrm{NaF}$ and $1 \%$ protease inhibitor cocktail (P8340; Sigma-Aldrich). After three freeze-thaw cycles in liquid nitrogen, we cleared the resulting cell lysates by a 10 -min $20000 \times g$ centrifugation at $4^{\circ} \mathrm{C}$.

Analysis of detergent soluble, detergent insoluble and urea soluble fractions. Cells $\left(10^{6}\right)$ were collected and washed twice in ice-cold PBS. Cell pellets were lysed in $100 \mu \mathrm{l}$ detergent-soluble fraction (DSF) buffer containing $10 \mathrm{mM}$ Tris- $\mathrm{HCl}(\mathrm{pH} 7.5), 1 \%$ Triton $\mathrm{X}-100,5 \mathrm{mM}$ EDTA and supplemented with $1 \mathrm{mM} \mathrm{Na}_{3} \mathrm{VO}_{4}, 50 \mathrm{mM} \mathrm{NaF}$ and $1 \%$ protease inhibitor cocktail. Insoluble material was recovered by centrifugation at $16000 \times g$ for $15 \mathrm{~min}$ at $4^{\circ} \mathrm{C}$. Pellets were then washed with supplemented DSF buffer, resuspended in $20 \mu$ l detergent-insoluble fraction (DIF) buffer containing $10 \mathrm{mM}$ Tris- $\mathrm{HCl}(\mathrm{pH} 7.5), 1 \% \mathrm{SDS}$ and supplemented with $1 \mathrm{mM} \mathrm{Na}_{3} \mathrm{VO}_{4}, 50 \mathrm{mM} \mathrm{NaF}$ and $1 \%$ protease inhibitor cocktail, incubated for $15 \mathrm{~min}$ at room temperature and for $2 \mathrm{~min}$ on ice, and sonicated following the addition of $50 \mu \mathrm{l}$ DSF buffer. After centrifugation at $16000 \times g$ for $5 \mathrm{~min}$ at $4^{\circ} \mathrm{C}$, we resuspended the remaining insoluble material in $10 \mu 150 \mathrm{mM}$ Tris- $\mathrm{HCl}(\mathrm{pH} 7)$ containing $8 \mathrm{M}$ urea and sonicated. Equal amounts of each fraction were heated for $15 \mathrm{~min}$ at $37^{\circ} \mathrm{C}$ in SDS-PAGE sample buffer and analyzed by SDS-PAGE.

Antibodies. Two peptides, an N-terminal specific to the mouse ASB2 $\beta$ isoform (ISTRGRQRAIGHEE) and a C-terminal common to ASB2 $\alpha$ and $\beta$ proteins (LAPERARLYEDRRS), were synthesized and coupled to keyhole limpet hemocyanin through a cysteine residue added to the $\mathrm{C}$ - or N-terminal amino acid of the peptides, respectively (MilleGen). Rabbit sera were collected 6 months after the initial injection (MilleGen). The serum raised against human ASB2 (1PNA) has been described previously. ${ }^{3}$ Primary antibodies were anti-MHC (F59), anti-elongin $B$ (FL-118), anti-Rbx2 (N15), anti-Erk2 (C-14), anti-myogenin (F5D; Santa Cruz Biotechnology), anti-troponin T (JLT-12; Sigma-Aldrich), anti-HA (1D1; Euromedex), anti-polyubiquitinylated proteins (FK1; BIOMOL), anti-elongin $\mathrm{C}$ (SIllp15; Transduction Laboratories), anti-FLNc (Kinasource), anti-Flag (F7425; Sigma-Aldrich) and anti-GFP (Rockland). The anti-human FLNa antiserum that cross-reacts with mouse FLNa has been described. ${ }^{40}$ Rabbit anti-FLNb and goat anti-FLNb (N-16) were purchased from Chemicon and Santa Cruz Biotechnology, respectively. Secondary antibody anti-mouse, anti-rabbit and anti-goat conjugates with HRP were from Jackson Laboratories.

Immunofluorescence. Cells were fixed in 4\% paraformaldehyde in PBS supplemented with $15 \mathrm{mM}$ sucrose and permeabilized with $0.1 \%$ Triton X-100. After blocking with $3 \%$ BSA in PBS, we performed immunostaining of cells using antibodies to FLNb from Chemicon in $1: 1000$ and to troponin $T$ in $1: 1000$. Secondary antibodies used were Alexa Fluor 546 or 488 coupled to goat anti-rabbit or goat anti-mouse (Invitrogen). For nuclear staining, we incubated fixed cells with $0.4 \mu \mathrm{M}$ DAPI for $5 \mathrm{~min}$ after secondary antibody incubation. Preparations were mounted in Mowiol (Calbiochem). 
Ubiquitylation assays. Recombinant baculoviruses encoding mASB2 $\beta$, $\mathrm{mASB} 2 \beta \mathrm{L} 595 \mathrm{~A}$ and Rbx2 were generated with the BacPAK baculovirus expression system (Clontech). In vitro ubiquitylation assays were carried out as described. ${ }^{5}$ FLNb ubiquitylation assays were performed using immunopurified FLNb-GFP as substrate in the presence of the NEDD8 machinery as described. ${ }^{6}$ Briefly, NIH3T3 cells were transfected for $24 \mathrm{~h}$ with FLNb-GFP expression vector. Anti-GFP antibodies immobilized onto Protein A Sepharose were added to the cell protein extract in a binding buffer adjusted to $20 \mathrm{mM}$ Tris- $\mathrm{HCl}(\mathrm{pH} 7.5), 150 \mathrm{mM}$ $\mathrm{NaCl}$ and $0.1 \% \mathrm{NP}-40$. After $2 \mathrm{~h}$ of incubation on ice and after three washes with binding buffer, we eluted proteins with $100 \mathrm{mM}$ phosphate buffer (pH 12.5) and buffered to $\mathrm{pH} 8.5$ for in vitro ubiquitylation assays. Reaction products were fractionated by SDS-PAGE and analyzed by immunoblotting with anti-GFP antibodies.

Acknowledgements. We thank $\mathrm{J}$ Dubrulle and $\mathrm{O}$ Pourquié for the chicken ASB2 probe and initial help with in situ hybridizations, M-A Bonnin for technical assistance with in situ hybridizations and V Mouly and the human cell culture platform from the Myology Institute in Paris for providing human primary myoblasts. We are grateful to $D$ Heard for the design of shRNAs directed against mouse ASB2 $\beta$. We thank Lucie Carrier for critical reading of the article. This work was supported by the Centre National de la Recherche Scientifique (CNRS), the Université de Toulouse, the Université Pierre et Marie Curie and by grants to DAC from the National Institutes of Health (GM068600 and HL089433), to CML from the Université Paul Sabatier and to PGL from the Agence Nationale de la Recherche (Programme Jeunes Chercheuses, Jeunes Chercheurs), the Association Française contre les Myopathies, the Association pour la Recherche sur le Cancer (Programme Equipe Nouvelle) and the Fondation pour la Recherche Médicale (Programme Installation d'une nouvelle équipe). NF Bello is supported by the Association Française contre les Myopathies. ML Heuzé was supported by a doctoral Allocation de Recherche du Ministère de la Recherche et des Technologies and by the Association pour la Recherche sur le Cancer.

1. Glickman $\mathrm{MH}$, Ciechanover $\mathrm{A}$. The ubiquitin-proteasome proteolytic pathway: destruction for the sake of construction. Physiol Rev 2002; 82: 373-428.

2. Hilton DJ, Richardson RT, Alexander WS, Viney EM, Willson TA, Sprigg NS et al. Twenty proteins containing a C-terminal SOCS box form five structural classes. Proc Natl Acad Sci USA 1998; 95: 114-119.

3. Guibal FC, Moog-Lutz C, Smolewski P, Di Gioia Y, Darzynkiewicz Z, Lutz PG et al. ASB-2 inhibits growth and promotes commitment in myeloid leukemia cells. J Biol Chem 2002; 277: 218-224.

4. Moog-Lutz C, Cave-Riant F, Guibal FC, Breau MA, Di Gioia Y, Couraud PO et al. JAML, a novel protein with characteristics of a junctional adhesion molecule, is induced during differentiation of myeloid leukemia cells. Blood 2003; 102: 3371-3378.

5. Heuze ML, Guibal FC, Banks CA, Conaway JW, Conaway RC, Cayre YE et al. ASB2 is an Elongin $\mathrm{BC}$-interacting protein that can assemble with Cullin 5 and $\mathrm{Rbx} 1$ to reconstitute an E3 ubiquitin ligase complex. J Biol Chem 2005; 280: 5468-5474

6. Heuze ML, Lamsoul I, Baldassarre M, Lad Y, Lévêque S, Razinia Z et al. ASB2 targets filamins A and B to proteasomal degradation. Blood 2008; 112: 5130-5140.

7. Lindon C, Montarras D, Pinset C. Cell cycle-regulated expression of the muscle determination factor Myf5 in proliferating myoblasts. J Cell Biol 1998; 140: 111-118.

8. Song $A$, Wang $Q$, Goebl MG, Harrington MA. Phosphorylation of nuclear MyoD is required for its rapid degradation. Mol Cell Biol 1998; 18: 4994-4999.

9. Sun L, Trausch-Azar JS, Ciechanover A, Schwartz AL. Ubiquitin-proteasome-mediated degradation, intracellular localization, and protein synthesis of MyoD and Id1 during muscle differentiation. J Biol Chem 2005; 280: 26448-26456.

10. Sun L, Trausch-Azar JS, Ciechanover A, Schwartz AL. E2A protein degradation by the ubiquitin-proteasome system is stage-dependent during muscle differentiation. Oncogene 2007; 26: 441-448.

11. Solomon V, Goldberg AL. Importance of the ATP-ubiquitin-proteasome pathway in the degradation of soluble and myofibrillar proteins in rabbit muscle extracts. $\mathrm{J}$ Biol Chem 1996; 271: 26690-26697.

12. Kudryashova $E$, Kudryashov D, Kramerova I, Spencer MJ. Trim32 is a ubiquitin ligase mutated in limb girdle muscular dystrophy type $2 \mathrm{H}$ that binds to skeletal muscle myosin and ubiquitinates actin. J Mol Biol 2005; 354: 413-424.

13. Witt SH, Granzier H, Witt CC, Labeit S. MURF-1 and MURF-2 target a specific subset of myofibrillar proteins redundantly: towards understanding MURF-dependent muscle ubiquitination. J Mol Biol 2005; 350: 713-722.
14. Zhao TJ, Yan YB, Liu Y, Zhou HM. The generation of the oxidized form of creatine kinase is a negative regulation on muscle creatine kinase. J Biol Chem 2007; 282: 12022-12029.

15. Pizon V, lakovenko A, Van Der Ven PF, Kelly R, Fatu C, Furst DO et al. Transient association of titin and myosin with microtubules in nascent myofibrils directed by the MURF2 RING-finger protein. J Cell Sci 2002; 115: 4469-4482.

16. McElhinny AS, Perry CN, Witt CC, Labeit S, Gregorio CC. Muscle-specific RING finger-2 (MURF-2) is important for microtubule, intermediate filament and sarcomeric M-line maintenance in striated muscle development. J Cell Sci 2004; 117: 3175-3188.

17. Spencer JA, Eliazer S, llaria Jr RL, Richardson JA, Olson EN. Regulation of microtubule dynamics and myogenic differentiation by MURF, a striated muscle RING-finger protein J Cell Biol 2000; 150: 771-784

18. Fielitz J, van Rooij E, Spencer JA, Shelton JM, Latif S, van der Nagel R et al. Loss of muscle-specific RING-finger 3 predisposes the heart to cardiac rupture after myocardial infarction. Proc Natl Acad Sci USA 2007; 104: 4377-4382.

19. Fielitz J, Kim MS, Shelton JM, Latif S, Spencer JA, Glass DJ et al. Myosin accumulation and striated muscle myopathy result from the loss of muscle RING finger 1 and 3. J Clin Invest 2007; 117: 2486-2495.

20. Tintignac LA, Lagirand J, Batonnet S, Sirri V, Leibovitch MP, Leibovitch SA. Degradation of MyoD mediated by the SCF (MAFbx) ubiquitin ligase. J Biol Chem 2005; 280: 2847-2856.

21. Nastasi T, Bongiovanni A, Campos Y, Mann L, Toy JN, Bostrom J et al. Ozz-E3, a musclespecific ubiquitin ligase, regulates beta-catenin degradation during myogenesis. Dev Cell 2004; 6: 269-282.

22. McDaneld TG, Hannon K, Moody DE. Ankyrin repeat and SOCS box protein 15 regulates protein synthesis in skeletal muscle. Am J Physiol Regul Integr Comp Physiol 2006; 290 R1672-R1682.

23. Li W, Wu G, Wan Y. The dual effects of Cdh1/APC in myogenesis. FASEB J 2007; 21 3606-3617

24. Kamura T, Maenaka K, Kotoshiba S, Matsumoto M, Kohda D, Conaway RC et al. VHL-box and SOCS-box domains determine binding specificity for Cul2-Rbx1 and Cul5-Rbx2 modules of ubiquitin ligases. Genes Dev 2004; 18: 3055-3065.

25. Young $P$, Deveraux $Q$, Beal RE, Pickart CM, Rechsteiner M. Characterization of two polyubiquitin binding sites in the $26 \mathrm{~S}$ protease subunit $5 \mathrm{a}$. J Biol Chem 1998; 273 5461-5467.

26. Hurley JH, Lee S, Prag G. Ubiquitin-binding domains. Biochem J 2006; 399: 361-372.

27. Flick K, Raasi S, Zhang H, Yen JL, Kaiser P. A ubiquitin-interacting motif protects polyubiquitinated Met4 from degradation by the 26S proteasome. Nat Cell Biol 2006; 8 : 509-515

28. van der Flier A, Kuikman I, Kramer D, Geerts D, Kreft M, Takafuta T et al. Different splice variants of filamin-B affect myogenesis, subcellular distribution, and determine binding to integrin [beta] subunits. J Cell Biol 2002; 156: 361-376.

29. Dedieu S, Poussard S, Mazeres G, Grise F, Dargelos E, Cottin P et al. Myoblast migration is regulated by calpain through its involvement in cell attachment and cytoskeletal organization. Exp Cell Res 2004; 292: 187-200.

30. Andres V, Walsh K. Myogenin expression, cell cycle withdrawal, and phenotypic differentiation are temporally separable events that precede cell fusion upon myogenesis. J Cell Biol 1996; 132: 657-666.

31. Menko AS, Boettiger D. Occupation of the extracellular matrix receptor, integrin, is a contro point for myogenic differentiation. Cell 1987; 51: 51-57.

32. Schwander M, Leu M, Stumm M, Dorchies OM, Ruegg UT, Schittny J et al. Beta1 integrins regulate myoblast fusion and sarcomere assembly. Dev Cell 2003; 4: 673-685.

33. Calderwood DA, Huttenlocher A, Kiosses WB, Rose DM, Woodside DG, Schwartz MA et al. Increased filamin binding to beta-integrin cytoplasmic domains inhibits cell migration Nat Cell Biol 2001; 3: 1060-1068.

34. McCarthy JJ, Andrews JL, McDearmon EL, Campbell KS, Barber BK, Miller BH et al. Identification of the circadian transcriptome in adult mouse skeletal muscle. Physio Genomics 2007; 31: 86-95.

35. Edom F, Mouly V, Barbet JP, Fiszman MY, Butler-Browne GS. Clones of human satellite cells can express in vitro both fast and slow myosin heavy chains. Dev Biol 1994; 164: 219-229.

36. Lad Y, Kiema T, Jiang P, Pentikainen OT, Coles CH, Campbell ID et al. Structure of three tandem filamin domains reveals auto-inhibition of ligand binding. EMBO J 2007; 26 3993-4004.

37. Chomczynski P, Sacchi N. Single-step method of RNA isolation by acid guanidinium thiocyanate-phenol-chloroform extraction. Anal Biochem 1987; 162: 156-159.

38. Lutz PG, Houzel-Charavel A, Moog-Lutz C, Cayre YE. Myeloblastin is an Myb target gene: mechanisms of regulation in myeloid leukemia cells growth-arrested by retinoic acid. Blood 2001; 97: 2449-2456.

39. Tozer S, Bonnin MA, Relaix F, Di Savino S, Garcia-Villalba P, Coumailleau P et al. Involvement of vessels and PDGFB in muscle splitting during chick limb development. Development 2007; 134: 2579-2591.

40. Kiema $\mathrm{T}$, Lad $\mathrm{Y}$, Jiang $\mathrm{P}$, Oxley CL, Baldassarre M, Wegener $\mathrm{KL}$ et al. The molecular basis of filamin binding to integrins and competition with talin. Mol Cell 2006; 21: 337-347. 